\title{
Propagation Model for Evaluating the Interference Between Neighboring Indoor Micro Operators
}

\author{
Kimmo Hiltunen and Marja Matinmikko-Blue \\ Centre for Wireless Communications \\ University of Oulu \\ Oulu, Finland \\ kimmo.hiltunen@oulu.fi; marja.matinmikko@oulu.fi
}

\begin{abstract}
Recently introduced 5G micro operator concept aims at providing high-quality mobile services in specific highdemand (indoor) locations to complement the coverage and capacity offered by the traditional mobile network operators (MNOs). For reliable service delivery, micro operators need local spectrum access rights with predefined levels of quality guarantees. This can be obtained via granting spectrum micro licenses, where the license areas can be the different buildings. To coordinate the interference between the micro operators themselves and potential incumbent spectrum users, accurate interference characterization between the involved systems is a necessity. This requires proper modeling of the radio wave propagation characteristics, which is currently missing for micro operator deployment scenarios in their target frequency bands. This paper introduces a new propagation model for evaluating the interference between neighboring micro operators located inside different buildings. The proposed model is based on a number of sub-models and components, and the basis for each of the components has been taken by selecting appropriate path loss models described in the literature. In all, the introduced propagation model is valid for deployments in different frequency bands, and for various types of buildings and outdoor environments surrounding the buildings with micro operator deployments.
\end{abstract} indoor;

Keywords—propagation; building-to-building; micro operator;

\section{INTRODUCTION}

Next generation mobile communication networks known as $5 \mathrm{G}$ are expected to enable reliable provisioning of high-quality wireless connectivity in specific high-demand areas such as campuses, transport hubs, hospitals, shopping malls and factories [1]. Mobile communication networks have been traditionally deployed by mobile network operators (MNO) in outdoors with country-wide exclusive licenses to provide wide area coverage. However, in case of $5 \mathrm{G}$ the importance of specific in-building networks will increase [2], in particular for deployments in the higher frequency bands (e.g., millimeter wave bands, 24-86 GHz). The challenge with MNOs separately deploying their in-building networks is that they do not scale when the number of buildings is increasing, either due to increased capacity requirements or higher center frequencies, and that the networks typically serve only the MNO's own customers. Instead of relying solely on the networks deployed by the MNOs, a more efficient solution could be to allow other

This work in "Micro-operator concept for boosting local service delivery in $5 \mathrm{G}(\mathrm{uO} 5 \mathrm{G})$ " project was funded by Business Finland. stakeholders, such as the venue owners, to deploy and operate their own in-building networks to satisfy local capacity and coverage needs [2].

The micro operator concept [3] with local spectrum micro licensing model [4][5] has been recently introduced for local 5G network deployments serving both its own restricted customer set and acting as neutral host for the customers of the overlying MNOs [6]. The introduction of potentially a large number of local indoor $5 \mathrm{G}$ micro operators calls for new local spectrum authorization models for novel deployment scenarios where different micro operators could deploy their local networks in neighboring buildings as discussed in [4]. For reliable service delivery, the micro operators would need to be issued local spectrum micro licenses [4][5], where some form of interference coordination is required between the different license holders and potential incumbent spectrum users. Interference coordination calls for accurate modeling of the different interference scenarios between the involved systems, which in turn requires proper modeling of the radio wave propagation in the specific frequency bands and for the new indoor deployments.

Both the propagation within a building and the propagation between indoor and outdoor environments have been widely discussed in the literature [7]-[13]. However, the topic of building-to-building propagation between indoor nodes located inside different buildings has received much less attention. One of the very few building-to-building propagation models known to the authors is the 3GPP dual-strip model described in [14]. The applicability of the 3GPP dual-strip model for the micro operator concept was evaluated in [15] through propagation loss measurements that identified the need for building-specific wall penetration losses and alternative models to characterize the outdoor propagation loss. Thus, the existing 3 GPP model has several weaknesses, which limit its use for characterizing the interference between neighboring micro operators. Therefore, this paper proposes a new type of model for building-to-building propagation that can be used to characterize the local micro operator network performance as well as the interference between separate micro operators in different frequency bands and deployment scenarios.

The rest of the paper is organized as follows. Section II discusses the usability of the 3GPP dual-strip model and proposes a new propagation model for micro operator deployments. Then, performance evaluation results are shown 


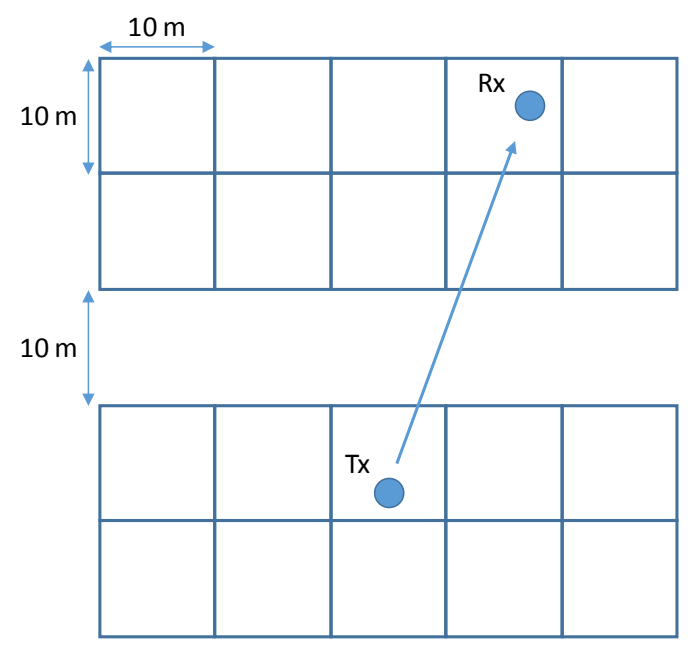

Fig. 1. 3GPP dual-strip deployment.

in Section III for a few example scenarios. Finally, some conclusions are drawn with suggestions for future enhancements in Section IV.

\section{Propagation Models Between Buildings}

When evaluating the impact of inter-operator interference on the performance of a local micro operator, path loss estimates both within the network of the victim micro operator inside the specific building (indoor propagation) and between the neighboring micro operators in separate buildings (building-to-building propagation) are required. This section presents first the 3GPP dual-strip propagation model [14] and discusses its feasibility for micro operator deployments. Then, a new propagation model is introduced, including sub-models for both the indoor and the building-to-building propagation.

\section{A. 3GPP Dual-Strip Model}

3GPP has defined the so-called dual-strip model in [14] for the estimation of path losses between indoor small cells and mobile terminals located either in the same or in different buildings in $2 \mathrm{GHz}$ and $3.5 \mathrm{GHz}$ frequency bands. The dualstrip model consists of two multi-floor buildings with apartments of size $10 \times 10$ meters. The buildings are separated by a distance of 10 meters, as illustrated in Fig. 1.

The model calculates the path loss between a base station and a mobile terminal located on the same floor as

$$
L_{d B}=38.46+20 \log _{10}(R)+0.5 d_{2 D, \text { indoor }}+q L_{i w}+\Delta\left(f_{c}\right)
$$

Furthermore, the path loss between a base station and a mobile terminal located in different building is calculated as

$$
\begin{aligned}
& L_{d B}=\max \left(15.3+37.6 \log _{10}(R), 38.46+20 \log _{10}(R)\right) \\
& +0.5 d_{2 D, \text { indoor }}+q L_{i w}+L_{o w 1}\left(f_{c}\right)+L_{o w 2}\left(f_{c}\right)+\Delta\left(f_{c}\right)
\end{aligned}
$$

In (1) and (2) $R$ is the distance in meters, $d_{2 D \text {,indoor }}$ is the distance traveled indoors, $q$ is the number of traversed inner walls, $L_{i w}$ is the loss per inner wall, and $L_{o w 1}\left(f_{c}\right)$ and $L_{o w 2}\left(f_{c}\right)$ are the outer wall losses of the two buildings as a function of the center frequency $f_{c}$. Based on the dual-strip model, $L_{o w}(2 \mathrm{GHz})$ $=20 \mathrm{dBm}, L_{o w}(3.5 \mathrm{GHz})=23 \mathrm{~dB}$ and $L_{i w}=5 \mathrm{~dB}$. Finally, in order to convert the path loss model from $2 \mathrm{GHz}$ to $3.5 \mathrm{GHz}$, a frequency correction factor $\Delta\left(f_{c}\right)$ equal to $4.8 \mathrm{~dB}$ is added $\left(\Delta(3.5 \mathrm{GHz})=20 \log _{10}(3.5 / 2)\right)$.

A closer examination of the details of the 3GPP dual-strip model reveals a number of weaknesses, which limit its usage for characterizing the interference between neighboring micro operators. These include the following:

- The model is tailored for $2 \mathrm{GHz}$ and $3.5 \mathrm{GHz}$ and is not valid for higher frequency bands envisaged for $5 \mathrm{G}$.

- The assumed building penetration model considers only one of the building walls (the one facing the neighboring building) and ignores the penetration through the other walls.

- The impact of building wall material on the building penetration loss is not taken into account. Instead, a fixed value equal to 20 or $23 \mathrm{~dB}$ is assumed, which will typically overestimate the path loss between the buildings, as shown by the measurement results in [15].

- The model ignores the impact of the angle of incidence on the building penetration loss.

- The outdoor path loss between the buildings is based on a macro cellular NLOS propagation model even at relatively short distances, which has been shown to overestimate the path losses between the buildings [15].

To overcome the limitations of the 3GPP dual-strip model, there is a need to develop a new building-to-building propagation model. The model proposed in the following subsection aims to consider the aspects listed above when modeling the path losses between the neighboring micro operators.

\section{B. Proposed Model for Micro Operator Deployments}

Similar to the 3GPP dual-strip model, also the proposed micro operator deployment model contains two neighboring buildings, see Fig. 2. The buildings are assumed to be located at a distance $D$ from each other. As a default, the model considers only one floor per building and instead of specifically modeling inner walls, the wall and furniture losses are assumed to be included in the distance-dependent indoor path loss. Finally, in addition to the sub-model for indoor propagation within the building, the overall model contains two optional sub-models for building-to-building propagation, depending on whether the buildings are in LOS or NLOS with each other.

The sub-models for indoor and building-to-building propagation are composed of different components and the basis for each of these components has been taken by selecting appropriate models described in the literature. One of the main requirements has also been that the center frequency $f_{c}$ is a parameter in the overall model, which means that the same model should be applicable for different frequency bands. In all, the sub-model for indoor propagation is based on the model 


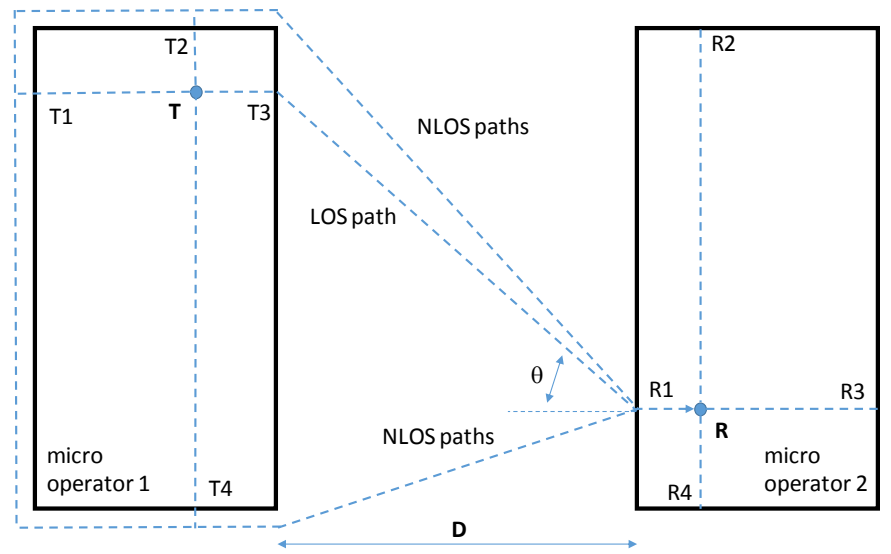

Fig. 2. Proposed building-to-building propagation model for micro operator deployment.

described in [10], while the sub-model for building-to-building propagation includes components for the recursive microcell model for outdoor propagation [16], NLOS outdoor propagation for microcells [10], general building penetration model [7], LOS building penetration model [7][11][13] including the impact of the angle of incidence, NLOS building penetration model [10] and the linear attenuation model for indoor propagation [7]. Furthermore, the building wall loss is assumed to depend both on the center frequency and on the wall material as defined in [10].

In the overall model, the coupling loss between a mobile terminal $m$ and a base station $b$ is calculated as

$$
C L_{m, b, d B}=L_{m, b, d B}-G_{B S, m, b, d B}-G_{M T, m, b, d B}+X_{m, b, d B}
$$

where $G_{B S, m, b}$ and $G_{M T, m, b}$ are the base station and mobile terminal antenna gains, respectively, and $X_{m, b}$ is a log-normally distributed random value modeling the impact of shadow fading. Next, the different sub-models and components forming the proposed model for the path loss $L_{m, b}$ are discussed in more detail.

The indoor propagation within a building is modeled using the 3GPP Indoor - Mixed Office propagation model as defined in [10]. The path loss model includes both a LOS $\left(L_{L O S}\right)$ and a NLOS $\left(L_{N L O S}\right)$ component, and the LOS probability $\left(P_{L O S}\right)$ is defined to decrease as a function of the distance between the base station and the mobile terminal. The standard deviation of the shadow fading is assumed to be equal to $3 \mathrm{~dB}$ (LOS) or 8 $\mathrm{dB}$ (NLOS) [10]. Furthermore, both the shadow fading and the LOS probability are spatially correlated, assuming correlation distances equal to $10 \mathrm{~m}$ and $6 \mathrm{~m}$ (shadow fading in LOS and NLOS), and $10 \mathrm{~m}$ (LOS probability) [10].

When it comes to modeling the path losses between the different buildings, four different sub-paths for each building, one through each building wall [7], are evaluated as shown in Fig. 2. Hence, for each link between a transmitter and a receiver, the total received power is calculated as a linear sum of the received powers from all the 16 different sub-paths. Each sub-path takes into account both the outdoor loss between the outer wall reference points (T1-T4 and R1-R4, having the same height as the corresponding indoor node), and the building penetration and indoor losses for both buildings. In all, the path loss per sub-path is calculated as

$$
L_{d B}\left(f_{c}\right)=L_{i n, 1}+L_{o w, 1}\left(f_{c}\right)+L_{o u t}\left(f_{c}\right)+L_{o w, 2}\left(f_{c}\right)+L_{i n, 2}
$$

In (4), $L_{i n}$ is the indoor loss, modeled as $L_{i n}=0.5 d_{2 D-i n}$, where $d_{2 D \text {-in }}$ is the two-dimensional distance between the indoor node and the outer wall reference point [10]. Parameter $L_{o w}$ models the building wall loss and it consists of two components: one that depends on the three-dimensional angle of incidence $\theta$ and the other that depends on the wall material and the center frequency $f_{c}$. Depending on the desired building penetration model (LOS or NLOS), $L_{o w}$ is calculated either as described in (5) [13] or in (6) [10].

$$
\begin{gathered}
L_{o w, L O S}=20(1-\cos \theta)^{2}+L_{\text {material }}\left(f_{c}\right) \\
L_{o w, N L O S}=5+L_{\text {material }}\left(f_{c}\right)
\end{gathered}
$$

If the buildings are in LOS with each other, (5) is applied for wall reference points T3 and R1, while (6) is applied for the other wall reference points. In case of a NLOS propagation condition between the buildings, (6) is applied for all wall reference points.

In higher frequency bands, the material characteristics of the building have a major impact on defining the penetration loss pattern, which means that the value of $L_{\text {material }}\left(f_{c}\right)$ can be significantly different for different buildings. Further discussion on the impact of $f_{c}$ on the wall penetration loss can be found for example in [10], [13] and [17]-[19]. Furthermore, the impact of different wall materials has been discussed in [10] and [17]-[23]. As an example, the penetration losses for standard multi-pane glass, infrared reflective (IRR) glass and concrete can be expressed as [10]:

$$
\begin{gathered}
L_{\text {glass }}=2+0.2 f_{c, G H z} \\
L_{\text {IRRglass }}=23+0.3 f_{c, G H z} \\
L_{\text {concrete }}=5+4 f_{c, G H z}
\end{gathered}
$$

Parameter $L_{\text {out }}$ in (4) is the outdoor path loss between the outer wall reference points. In case of a LOS path, i.e., the subpath between wall reference points T3 and R1 as shown in Fig. $2, L_{\text {out }}$ is based on a free space propagation model, and the applied distance is a sum of the outdoor and indoor distances [7][11][15]. In case of a NLOS path, $L_{\text {out }}$ considers only the outdoor path between the outer wall reference points, and the applied propagation model depends on whether the buildings are in LOS or NLOS with each other.

If the buildings are in LOS with each other, $L_{\text {out }}$ for the NLOS paths is based on the recursive microcell model [16]: going around either one or two building corners as illustrated in Fig. 2, and assuming a breakpoint for the path loss exponent at $300 \mathrm{~m}$ [16]. However, if the buildings are in NLOS with each 
TABLE I.

ASSUMED PARAMETER VALUES

\begin{tabular}{|c|c|}
\hline Parameter & Value \\
\hline Center frequency $\left(f_{c}\right)$ & $3.5 \mathrm{GHz}, 26 \mathrm{GHz}$ \\
\hline Base station transmision power & $24 \mathrm{dBm}$ \\
\hline $\begin{array}{l}\text { Mobile terminal receiver noise } \\
\text { power }\end{array}$ & $-92 \mathrm{dBm}$ \\
\hline Base station antenna gain & $5 \mathrm{dBi}$ (omnidirectional) \\
\hline Mobile terminal antenna gain & $0 \mathrm{dBi}$ (omnidirectional) \\
\hline Antenna height (above floor) & $3 \mathrm{~m}(\mathrm{BS}), 1 \mathrm{~m}(\mathrm{MT})$ \\
\hline Building wall losses $\left(L_{\text {material }}\right)$ & $\begin{array}{l}\text { 7.7 dB (low-loss wall, } 3.5 \mathrm{GHz} \text { ) } \\
12.4 \mathrm{~dB} \text { (low-loss wall, } 26 \mathrm{GHz} \text { ) } \\
21.8 \mathrm{~dB} \text { (high-loss wall, } 3.5 \mathrm{GHz} \text { ) } \\
32.3 \mathrm{~dB} \text { (high-loss wall, } 26 \mathrm{GHz} \text { ) }\end{array}$ \\
\hline
\end{tabular}

other, $L_{\text {out }}$ is based on the $3 G P P$ Urban Micro - Street Canyon propagation model defined in [10]: A weighted average of the LOS and NLOS path loss components $\left(L_{o u t}=P_{L O S} L_{L O S}+(1-\right.$ $\left.\left.P_{L O S}\right) L_{N L O S}\right)$ is applied for the sub-path between reference points T3 and R1, while only the NLOS path loss component ( $\left.L_{N L O S}\right)$ is applied for all the other sub-paths.

Finally, when it comes to the shadow fading model for the building-to-building propagation, standard deviation equal to 6 $\mathrm{dB}$ (LOS) or $8 \mathrm{~dB}$ (NLOS), and correlation distance equal to 10 $\mathrm{m}$ (LOS) or $13 \mathrm{~m}$ (NLOS) are assumed [10].

\section{EXAMPLE SCENARIOS}

In this section the proposed path loss model is visualized for a few example scenarios in $3.5 \mathrm{GHz}$ and $26 \mathrm{GHz}$ frequency bands, where the level of downlink interference between two neighboring micro operators is evaluated. The $3.5 \mathrm{GHz}$ frequency band is of special interest due to the recent development of the Citizens Broadband Radio Service (CBRS) in the US [24]. Also in Europe, the $3.4-3.8 \mathrm{GHz}$ band is seen as the first primary band for $5 \mathrm{G}$ [25]. Furthermore, the $26 \mathrm{GHz}$ band is seen as the pioneer band in Europe above $24 \mathrm{GHz}$ to give ultra-high capacity for innovative new services, enabling new business models and sectors of the economy [25].

The evaluated network layout consists of two equally-sized buildings $(50 \times 120 \mathrm{~m}$, based on the indoor deployment scenario defined in [10]), which are assumed to be in LOS with each other. Micro operator $1(\mathrm{uO} 1)$ is assumed to be serving users within the first building, while micro operator $2(\mathrm{uO} 2)$ is serving users within the second building. The micro operator networks are assumed to be located on the same floor level, i.e., the antenna heights (above the ground level) are assumed to be the same. It is further assumed that micro operator $\mathrm{uO} 1$ has deployed 12 pico base stations per floor as described in [10], while micro operator $\mathrm{uO} 2$ has deployed only one pico base station in the middle of the floor.

The assumed parameter values are listed in Table I. The analysis assumes also that the interfering network is fullyloaded, i.e., all base stations are transmitting at the maximum power. At the same time, the victim network is assumed to be serving only one user. Furthermore, the user is assumed to be
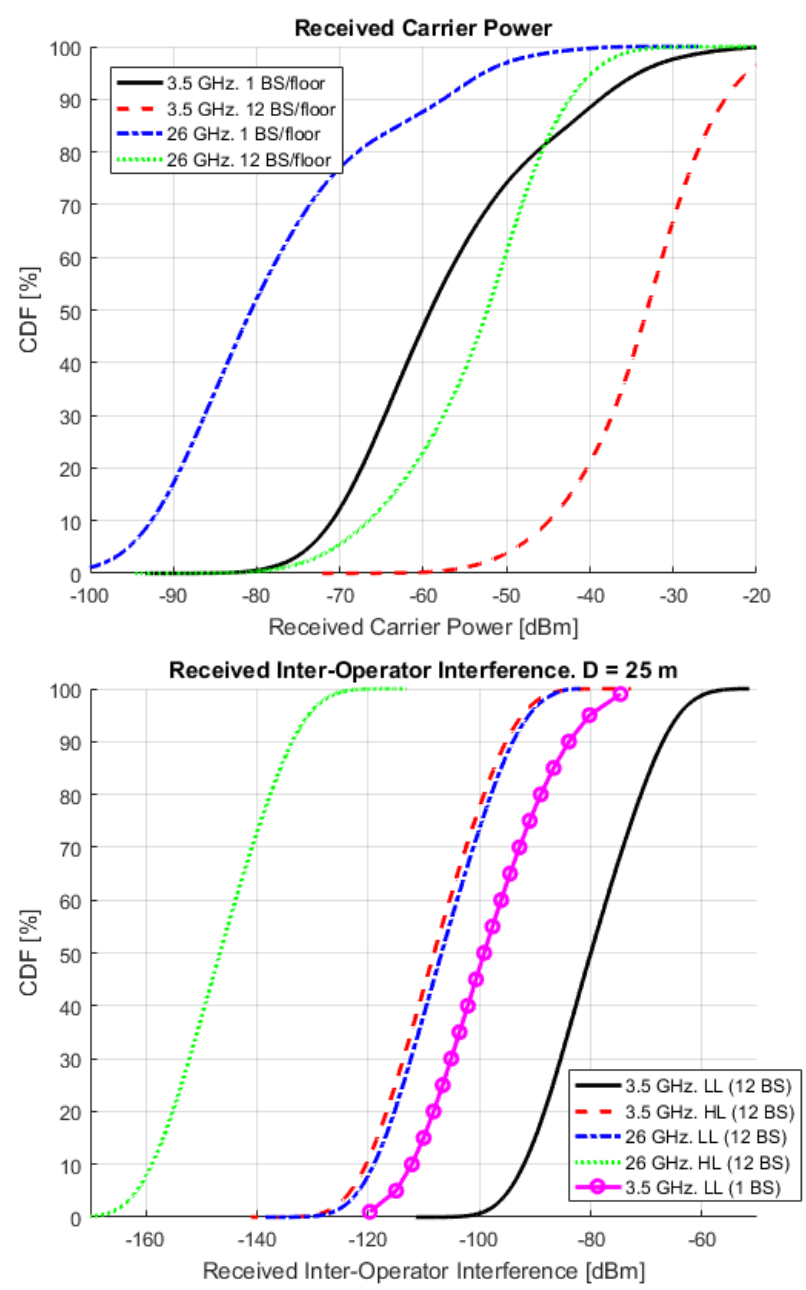

Fig. 3. Distributions for the received carrier power and the received interoperator interference power.

served by closest base station. Finally, the applied building wall losses have been calculated based on the average building wall loss model in [10]: low-loss (LL) wall assumes old buildings with $30 \%$ multi-pane windows and $70 \%$ concrete, while the high-loss (HL) wall assumes modern buildings with $70 \%$ IRR glass and $30 \%$ concrete.

To start with, Fig. 3 presents the distributions of the received carrier power and the received inter-operator interference for both frequency bands and the different building wall losses, assuming that the buildings are located at a distance of $25 \mathrm{~m}$ from each other. The results indicate that even a single pico base station at $3.5 \mathrm{GHz}$ deployed in the middle of the floor is able to provide received carrier power levels better than $-80 \mathrm{dBm}$ throughout the desired coverage area. At the same time, the resulting level of the inter-operator interference within the neighboring building is less than $-72 \mathrm{dBm}$. In case of the denser deployment with 12 pico base stations per floor, the coverage is considerably better, with received carrier power levels higher than $-57 \mathrm{dBm}$, but at the same time the level of the peak inter-operator interference is increased to $-56 \mathrm{dBm}$. Finally, in case of the high-loss building walls, the level of the 

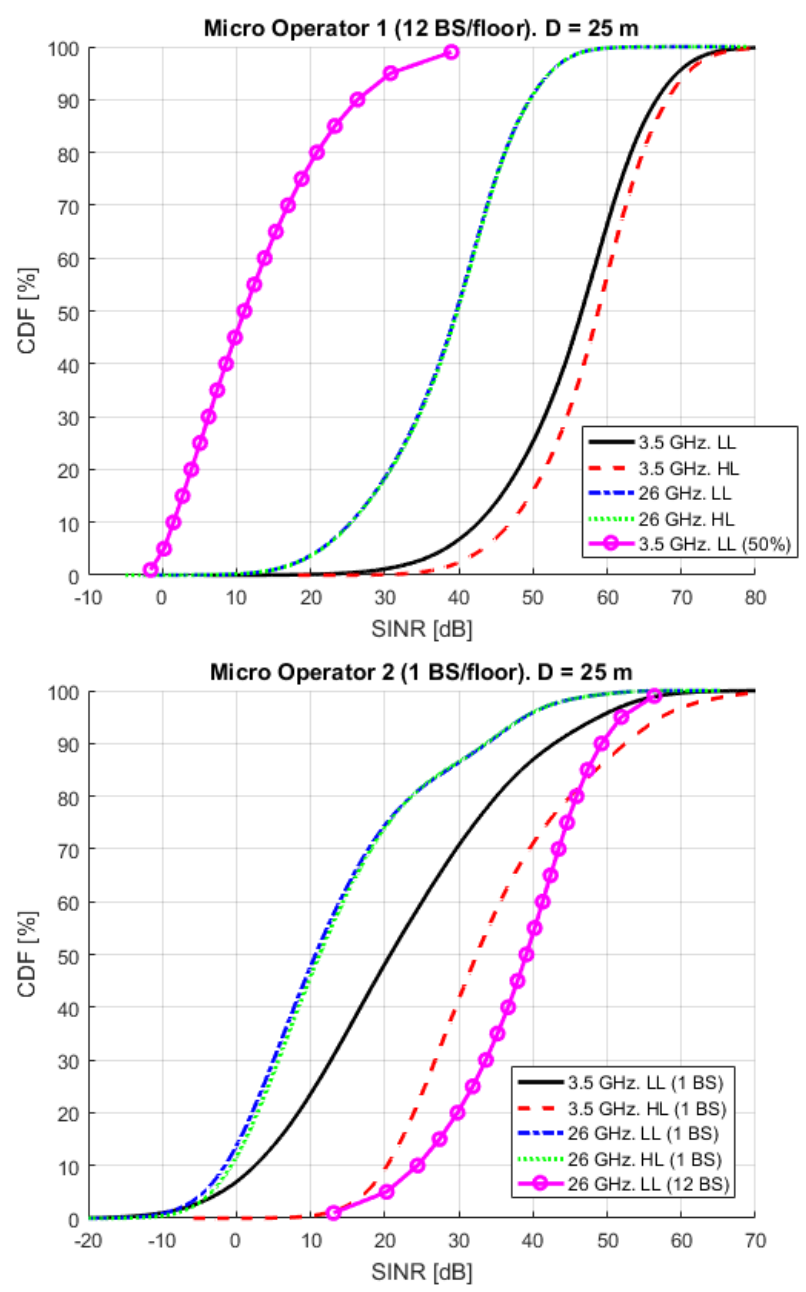

Fig. 4. Distributions for the received signal-to-interference-and-noise-ratio.

inter-operator interference becomes $28 \mathrm{~dB}$ lower compared to the low-loss walls.

When the center frequency is increased to $26 \mathrm{GHz}$, the received carrier power is reduced by 17 or $22 \mathrm{~dB}$, depending on whether the mobile terminal is in LOS or NLOS with the serving base station. This calls for either a much denser deployment of base stations or the use of more advanced antennas with beamforming capabilities in order to achieve a similar cell edge coverage than with $3.5 \mathrm{GHz}$. The increased center frequency will result also in a considerably lower interference towards to the neighboring micro operators, both due to increased building wall losses (an additional loss of 4.7 $\mathrm{dB} /$ wall for $\mathrm{LL}$ and $10.5 \mathrm{~dB} /$ wall for $\mathrm{HL}$ ) and increased outdoor path loss (an additional loss of $17.4 \mathrm{~dB}$ ). However, a densified deployment of base stations or the use of advanced antennas to improve the coverage will increase the interference towards the neighboring systems as well.

The corresponding signal-to-interference-and-noise-ratios (SINR) are presented in Fig. 4. In the figures, the own (victim) network is assumed to be serving only one user, while the other (interfering) network is assumed to be fully-loaded. In case of $\mathrm{uO} 2$, the performance of the sparse network deployment with

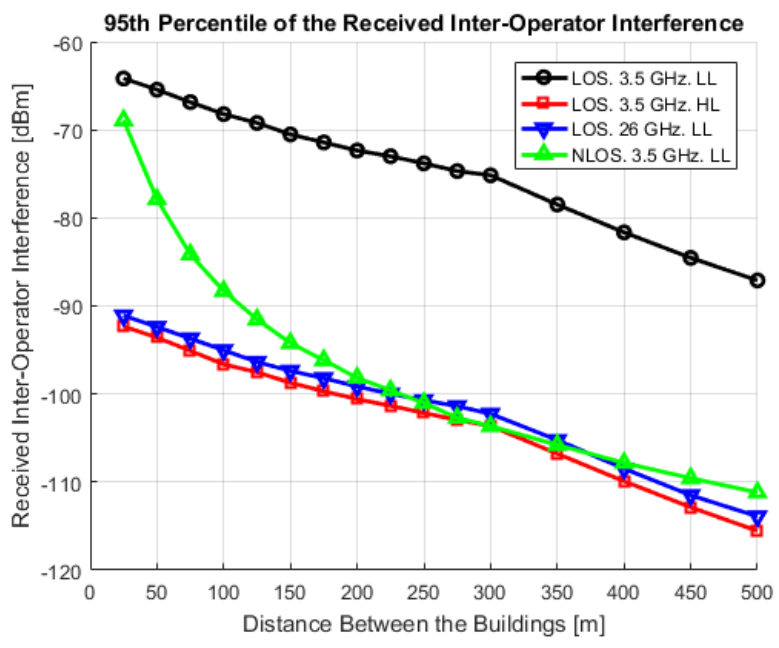

Fig. 5. $95^{\text {th }}$ percentile of the received inter-operator interference within the $\mathrm{uO} 2$ building as a function of the distance between the buildings.

one pico base station is quite sensitive to any external interference. For example, in case of the deployment with lowloss building walls in the $3.5 \mathrm{GHz}$ frequency band, the worst $5^{\text {th }}$ percentile of the users experience a SINR equal to $-2 \mathrm{~dB}$, while the median is at $21 \mathrm{~dB}$. In case of high-loss walls the level of inter-operator interference is reduced, which considerably improves the SINR values (18 dB (worst 5\%) and $32 \mathrm{~dB}$ (median)). When the center frequency is increased to 26 $\mathrm{GHz}$, both the received carrier power and the inter-operator interference levels become lower. In all, the SINR is reduced to $-4 \mathrm{~dB}$ (worst 5\%) and $11 \mathrm{~dB}$ (median). However, the coverage can be improved by densifying the network. For example, with 12 base stations per floor the SINR becomes equal to $20 \mathrm{~dB}$ (worst 5\%) and $39 \mathrm{~dB}$ (median).

In case of $\mathrm{uO} 1$, a dense indoor network with 12 base stations per floor is assumed, and therefore the impact of interoperator interference is much smaller compared to $\mathrm{uO} 2$. Due to both a higher received carrier power and a lower level of interoperator interference, the SINR values are much higher compared to $\mathrm{uO} 2$. Now, the worst $5^{\text {th }}$ percentile of users experience a SINR equal to $38 \mathrm{~dB}$ (LL) or $43 \mathrm{~dB}$ (HL), while the median is at $57 \mathrm{~dB}$ (LL) or $59 \mathrm{~dB}$ (HL). Similar to uO2, the center frequency has a large negative impact on the SINR coverage. However, the results demonstrate also that the impact of any intra-operator interference (assuming a 50\% load within the uO1 network as an example) is much higher than the impact of inter-operator interference.

Finally, Fig. 5 illustrates the $95^{\text {th }}$ percentile of the received inter-operator interference within the $\mathrm{uO} 2$ building as a function of the distance between the buildings, assuming different frequency bands, different building wall losses and different outdoor propagation models. Furthermore, the interfering $\mathrm{uO} 1$ network is assumed to be fully-loaded. As can be seen, the LOS deployment with low-loss building walls in the $3.5 \mathrm{GHz}$ frequency band results in by far the highest level of inter-operator interference. For example, if a threshold equal to $-85 \mathrm{dBm}$ is assumed for the level of the received interoperator interference, the required minimum distance between 
the buildings becomes equal to $460 \mathrm{~m}$. In case of NLOS propagation at $3.5 \mathrm{GHz}$, the required minimum distance is reduced to $80 \mathrm{~m}$. Finally, in case of high-loss building walls, or with center frequency equal to $26 \mathrm{GHz}$, the level of the worst case inter-operator interference becomes low enough to allow the micro operator buildings to be located right next to each other.

\section{CONCLUSIONS}

Sufficiently accurate propagation models are critical for the deployment of $5 \mathrm{G}$ networks that will increasingly operate in indoor locations and higher frequency bands. In particular, new path loss models are required to evaluate both the performance of the new local micro operator networks, as well as the level and the impact of interference between the micro operators and potential incumbent spectrum users in the band. In this paper, a new propagation model has been introduced to characterize the path loss between indoor networks deployed by different local micro operators in adjacent buildings in the same band. The model includes sub-models for both the indoor propagation within the micro operator network and the building-to-building propagation between the micro operators.

As visualized by the example scenarios, the introduced propagation model supports the use of different frequency bands, different building wall materials and different propagation conditions between the neighboring buildings. For example, the obtained results demonstrate how the network coverage and the link quality are reduced as the center frequency is increased, even though the level of the interoperator interference becomes considerably lower. In the future, the plan is to enhance the model with the support of three-dimensional beamforming for the base station antennas, three-dimensional indoor propagation model including a model for the floor penetration loss, and models for outdoor-to-indoor and indoor-to-outdoor propagation to enable coexistence evaluations with outdoor systems. Furthermore, the aim is to verify the propagation model with measurements also within the higher frequency bands.

\section{REFERENCES}

[1] The 5G Infrastructure Public Private Partnership (5GPPP), "5G innovations for new business opportunities", 5GPPP, Brussels, Tech. Rep., 2017.

[2] J. Zander, "Beyond the ultra-dense barrier: Paradigm shifts on the road beyond 1000x wireless capacity", IEEE Wireless Communications, vol. 24, no. 3, pp. 96-102, Jan. 2017.

[3] M. Matinmikko, M. Latva-aho, P. Ahokangas, S. Yrjölä, and T. Koivumäki, "Micro operators to boost local service delivery in $5 \mathrm{G}$ ", Wireless Personal Communications, vol. 95, no. 1, pp. 69-82, Jul. 2017.

[4] M. Matinmikko, A. Roivainen, M. Latva-aho, and K. Hiltunen, "Interference study of micro licensing for $5 \mathrm{G}$ micro operator small cell deployments", in Proc. Conference on Cognitive Radio Oriented Wireless Networks and Communications (CrownCom), Lisboa, Portugal, Sep. 2017.

[5] M. Matinmikko, M. Latva-aho, P. Ahokangas, and V. Seppänen, "On regulations for 5G: Micro licensing for locally operated networks", Telecommunications Policy, in press.
[6] P. Ahokangas, M. Matinmikko, S. Yrjölä \& I. Atkova, "Disruptive revenue models for future micro operator driven mobile business ecosystem", in Proc. The 24th Nordic Academy of Management Conference (NFF), Bodo, Norway, August 2017.

[7] E. Damasso, and L. M. Correia (ed.), "COST 231 Final Report: Digital mobile radio towards future generation systems", Tech. Rep., 1999.

[8] P. Heino (ed.), "D5.3: WINNER+ Final Channel Models", Tech. Rep., Jun. 2010.

[9] K. Haneda, et al., "Indoor 5G 3GPP-like Channel Models for Office and Shopping Mall Environments", in Proc. IEEE International Conference on Communications (ICC), Kuala Lumpur, Malaysia, May 2016.

[10] 3GPP, "Study on channel model for frequencies from $0.5 \mathrm{GHz}$ to 100 GHz", 3GPP TR 38.901, V14.1.1, Jul 2017.

[11] J.-E. Berg, "Building penetration loss along urban street microcells", in Proc. IEEE International Symposium on Personal, Indoor and Mobile Radio Communications (PIMRC), Taipei, Taiwan, Oct. 1996.

[12] G. Durgin, T. Rappaport, and H. Xu, "Measurements and models for radio path loss and penetration loss in and around homes and trees at $5.85 \mathrm{GHz}$ ", IEEE Transactions on Communications, vol. 46, no. 11, pp. 1484-1496, 1998

[13] E. Semaan, F. Harrysson, A. Furuskär, and H. Asplund, "Outdoor-toindoor coverage in high frequency bands", in Proc. IEEE Globecom 2014 Workshop - Mobile Communications in Higher Frequency Bands, Austin, TX, USA, Dec. 2014.

[14] 3GPP, "Small cell enhancements for E-UTRA and E-UTRAN Physical layer aspects", 3GPP TR 36.872, V12.1.0, Dec. 2013.

[15] J. Lehtomäki, R. Vuohtoniemi, M. Matinmikko-Blue, and K. Hiltunen, "Building-to-building propagation loss measurements at $3.5 \mathrm{GHz}$ with application to micro operators", accepted for publication at IEEE Wireless Communications and Networking Conference (WCNC) 2018.

[16] J.-E. Berg, "A recursive method for street microcell path loss calculations", in Proc. IEEE International Symposium on Personal, Indoor and Mobile Radio Communications (PIMRC), Toronto, Canada, Sep. 1995.

[17] Y. Du, et al., "Measurement and modeling of penetration loss in the range from $2 \mathrm{GHz}$ to $74 \mathrm{GHz}$ ", in Proc. IEEE Globecom Workshops (GC Wkshps), Washington, DC, USA, Dec. 2016.

[18] C. A. L. Diakhate, J.-M. Conrat, J.-C. Cousin, and A. Sibille, "Millimeter-wave outdoor-to-indoor channel measurements at 3,10,17 and $60 \mathrm{GHz}$ ", in Proc. European Conference on Antennas and Propagation (EuCAP), Paris, France, Mar. 2017.

[19] ITU, "ITU-R P.2346-2: Compilation of measurement data relating to building entry loss", Tech. Rep. ITU-R P.2346-2, 2017.

[20] H. Zhao, et al., "28 GHz millimeter wave cellular communication measurements for reflection and penetration loss in and around buildings in New York City", in Proc. IEEE International Conference on Communications (ICC), Budapest, Hungary, Jun. 2013.

[21] J. Lu, D. Steinbach, P. Cabrol, P. Pietraski, and R. V. Pragada, "Propagation characterization of an office building in the $60 \mathrm{GHz}$ band", in Proc. European Conference on Antennas and Propagation (EuCAP), The Hague, The Netherlands, Apr. 2014

[22] C. Larsson, F. Harrysson, B.-E. Olsson, and J.-E. Berg, "An outdoor-toindoor propagation scenario at $28 \mathrm{GHz}$, in Proc. European Conference on Antennas and Propagation (EuCAP), The Hague, The Netherlands, Apr. 2014.

[23] P. Ökvist, et al., " $15 \mathrm{GHz}$ propagation properties assessed with $5 \mathrm{G}$ radio access prototype", in Proc. IEEE International Symposium on Personal, Indoor and Mobile Radio Communications (PIMRC) - Workshop on 5G Channel Measurements and Modeling, Hong Kong, China, Aug. 2015.

[24] FCC, "Amendment of the commissions rules with regard to commercial operations in the $3550-3650 \mathrm{MHz}$ band, order on reconsideration and second report and order, Doc.No 12-354", FCC, Tech. Rep., May 2016.

[25] European Commission, Radio Spectrum Policy Group, "Strategic spectrum roadmap towards 5G for Europe", Draft RSPG Second Opinion on 5G networks, RSPG17-034, Brussels, Nov. 2017. 